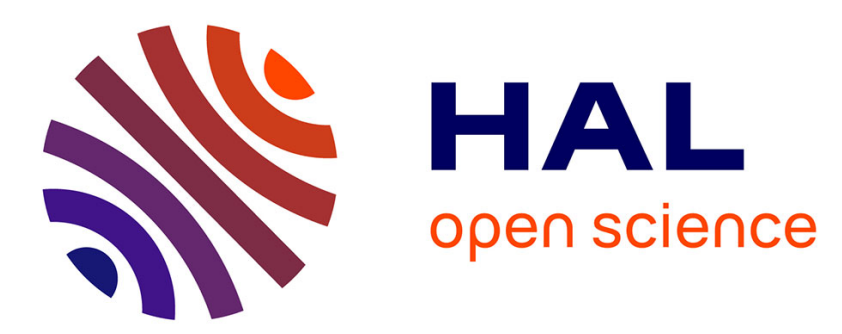

\title{
An Overlapping Nonmatching Grid Mortar Element Method for Maxwell's Equations
}

Alexandra Christophe, Laurent Santandrea, Francesca Rapetti, Guillaume Krebs, Yann Le Bihan

\section{To cite this version:}

Alexandra Christophe, Laurent Santandrea, Francesca Rapetti, Guillaume Krebs, Yann Le Bihan. An Overlapping Nonmatching Grid Mortar Element Method for Maxwell's Equations. IEEE Transactions on Magnetics, 2014, 50 (2), pp.Article 7010004. 10.1109/TMAG.2013.2282034 . hal-01093403

\section{HAL Id: hal-01093403 \\ https://hal-centralesupelec.archives-ouvertes.fr/hal-01093403}

Submitted on 11 Mar 2020

HAL is a multi-disciplinary open access archive for the deposit and dissemination of scientific research documents, whether they are published or not. The documents may come from teaching and research institutions in France or abroad, or from public or private research centers.
L'archive ouverte pluridisciplinaire HAL, est destinée au dépôt et à la diffusion de documents scientifiques de niveau recherche, publiés ou non, émanant des établissements d'enseignement et de recherche français ou étrangers, des laboratoires publics ou privés. 


\title{
An Overlapping Nonmatching Grid Mortar Element Method for Maxwell's Equations
}

\author{
Alexandra Christophe ${ }^{1}$, Laurent Santandrea ${ }^{1}$, Francesca Rapetti ${ }^{2}$, Guillaume Krebs ${ }^{1}$, and Yann Le Bihan ${ }^{1}$ \\ ${ }^{1}$ Laboratoire de Génie Electrique de Paris, University of Paris-Sud, UPMC Univ., Gif-sur-Yvette Cedex 91192, France \\ ${ }^{2}$ University of Nice Sophia Antipolis, CNRS, Lab. J.-A Dieudonné, Nice 06100, France
}

In this paper, a new finite element mortar approach with moving nonmatching overlapping grids is introduced. The bidirectional transfer of information between the fixed and moving subdomains is realized for each new position of the moving part. Two numerical examples are presented to support the theory: 1) an electrostatic problem with known solution, to state the optimality of the method and 2) an eddy current nondestructive testing configuration, to underline the flexibility and efficiency of the proposed approach.

Index Terms-FEMs, interface conditions, Maxwell equations, nondestructive testing, overlapping meshes.

\section{INTRODUCTION}

$\mathbf{T}$ HE modelization in eddy current (EC) nondestructive testing (NDT) aims at reproducing the interaction between an EC sensor and the inspected conductor to characterize the material or to localize possible defects in the latter. The FEM is frequently used in this context as well suited to treat problems with complex geometries while keeping a simplicity in the implementation. However, in NDT, the modelization has to be realized for different positions of the sensor, thus requiring, generally, a global remeshing of the domain. In the last two decades, different techniques to consider the displacement of a sensor without having to remesh the whole computational domain [1] [4] have been studied. The mortar element method [5] (MEM), a variational nonconforming domain decomposition approach, offers attractive advantages in terms of flexibility and accuracy. In its original version for non-overlapping subdomains, the information is transferred through the skeleton of the decomposition using a suitable $L^{2}$-projection of the field trace [6], [7]. Recently, a MEM with overlapping subdomains but unidirectional information transfer from the master subdomain to the slave one has been proposed [8], particularizing the general approach first presented in [9]. The MEM applied in [8] is suitably modified to deal with bidirectional information transfer between the overlapping subdomains in 2-D cases. This paper is organized as follows. An EC problem is presented in its weak domain decomposition version. The new overlapping nonmatching grid MEM is then described. The accuracy of this new approach is tested on a simple electrostatic problem, for which the analytical solution is well known. The method is then applied to solve an EC NDT problem to show its applicability.

Manuscript received June 28, 2013; revised August 12, 2013 and September 9, 2013; accepted September 12, 2013. Date of current version February 21, 2014. Corresponding author: A. Christophe (e-mail: alexandra.christophe@1gep.supelec.fr).

Color versions of one or more of the figures in this paper are available online at http://ieeexplore.ieee.org.

Digital Object Identifier 10.1109/TMAG.2013.2282034
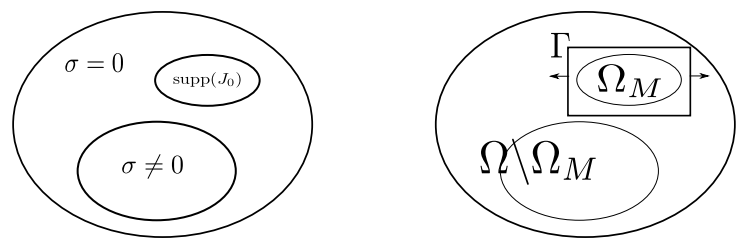

Fig. 1. Domain $\Omega$ for an EC case (left). Domain decomposition into a moving $\Omega_{M}$ and a fixed $\Omega$ subdomain (right).

\section{Magnetodynamic Formulation}

The time-harmonic EC equations in a domain $\Omega$ read

$$
\operatorname{curl} \mathbf{H}=\mathbf{J}, \quad \operatorname{curl} \mathbf{E}=-i \omega \mathbf{B}, \quad \operatorname{div} \mathbf{B}=0
$$

where $\mathbf{H}, \mathbf{B}, \mathbf{J}$, and $\mathbf{E}$ denote the magnetic field, the magnetic flux density, the current density, and the electric field, respectively. The constitutive laws $\mathbf{B}=\mu \mathbf{H}, \mathbf{J}=\mathbf{J}_{\mathbf{0}}+\sigma \mathbf{E}$, with the magnetic permeability $\mu$, the electric conductivity $\sigma$, and the source current $\mathbf{J}_{\mathbf{0}}$ distributed in $\Omega$, as shown in Fig. 1 left, are added together with boundary conditions to close the system (1). In this paper, a 2-D case is examined, where $\mathbf{B}$ lies in the $z=0$ plane. A magnetic vector potential $\mathbf{A}$ is defined such that $\mathbf{B}=\mathbf{c u r l} \mathbf{A}$ and $\mathbf{E}=-i \omega \mathbf{A}$; it is thus perpendicular to the $z=0$ plane and we denote by $A$ its (unique) unknown $z$-component.

Let us consider the domain $\Omega$ where an EC problem (1) is solved. In a domain decomposition approach, we separate the fixed and the moving subdomains in $\Omega$. The moving part, $\Omega_{M}$, contains the coil supporting $\mathbf{J}_{\mathbf{0}}$ with a layer of air and it is supposed to move over the whole domain $\Omega$ that includes some conducting and nonconducting parts (Fig. 1 right). In terms of scalar potential ( $A$ in fixed and $A_{M}$ in moving subdomain), the problem (1) is reduced to solve magnetostatic or magnetodynamic equations in each subdomain with $A-A_{M}=0$, $\mu_{M}^{-1} \partial_{n} A_{M}-\mu^{-1} \partial_{n} A=0$ as interface conditions on $\Gamma=\partial \Omega_{M}$ and homogeneous Neumann conditions on $\partial \Omega$. We remark that the moving part $\Omega_{M}$ is in reality kept fixed during each NDT simulation, so there is no influence of the motion of $\Omega_{M}$ on the distribution of EC in $\Omega$. Indeed, the term «moving» 

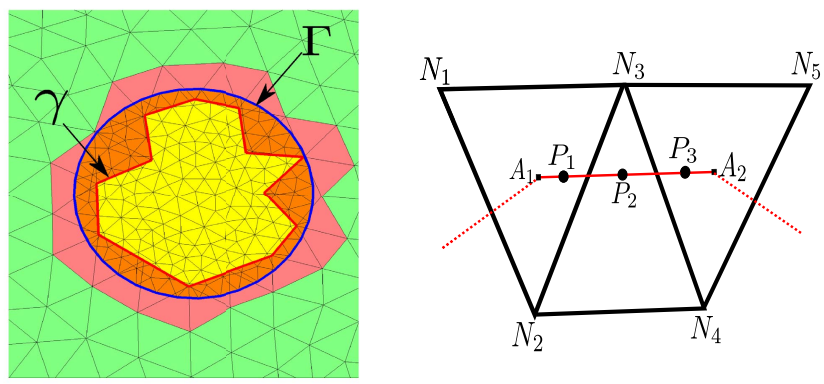

Fig. 2. Example of overlapping meshes (left) and computation of matrix $\mathrm{D}$ for an edge $\left[A_{1}, A_{2}\right]$ (right).

here denotes the fact that we will keep the same mesh in $\Omega_{M}$ independently from its position over $\Omega$, to avoid recreating the mesh for each new NDT simulation. In the present approach, the field source is in the moving part, differently from the application in [8].

The associated variational problem reads find $\left(A, A_{M}\right) \in H^{1}(\Omega) \times H_{A}^{1}\left(\Omega_{M}\right)$ such as

$$
\begin{aligned}
& \int_{\Omega_{M}} \mu^{-1} \nabla A_{M} \cdot \nabla A_{M}^{\prime}=\int_{\operatorname{supp} J_{0}} J_{0} A_{M}^{\prime} \quad \forall A_{M}^{\prime} \in H_{A}^{1}\left(\Omega_{M}\right) \\
& \int_{\Omega \backslash \Omega_{M}} i \sigma \omega A A^{\prime}+\int_{\Omega} \mu^{-1} \nabla A . \nabla A^{\prime}=0 \quad \forall A^{\prime} \in H^{1}(\Omega)
\end{aligned}
$$

where $H_{A_{M}}^{1}\left(\Omega_{M}\right)=\left\{A \in H^{1}\left(\Omega_{M}\right), A=A_{M}\right.$ on $\left.\Gamma\right\}$. It can be shown that there is a unique solution for (2). The continuous case gives $\left.A\right|_{\Gamma}=\left.A_{M}\right|_{\Gamma}$ but the main difficulty arises with the discrete case.

\section{Mortar Element Method}

Two triangulations $\mathcal{T}_{F}$ and $\mathcal{T}_{M}$ are applied depending on the fixed and moving subdomains, respectively. These discretizations are non matching grids and completely independent in the overlapping region. The exchange from $\Omega$ to $\Omega_{M}$ is realized on the interface $\Gamma$ (3) [8]. The information from moving to fixed subdomains uses an interface chosen as a boundary (called $\gamma$ ) of the elements of $\Omega$ overlapped by $\Gamma$ Fig. 2 (left) (4)

$$
\begin{aligned}
C A_{M \mid \Gamma} & =D A \\
E A_{\mid \gamma} & =H A_{M}
\end{aligned}
$$

according to the mortar method. The coupling matrices are described, on the edges $e$ of concerned interfaces, as

$$
\begin{aligned}
& C(i, j)=\int_{\mathrm{e} \in \Gamma} \psi_{j} \psi_{i} \quad D(i, k)=\int_{\mathrm{e} \in \Gamma} \phi_{k} \psi_{i} \\
& E(i, j)=\int_{\mathrm{e} \in \gamma} \phi_{j} \phi_{i} \quad H(i, k)=\int_{\mathrm{e} \in \gamma} \psi_{k} \phi_{i}
\end{aligned}
$$

where the functions $\phi_{i}$ and $\psi_{i}$ are defined on the discretizations of $\Omega$ and $\Omega_{M}$, respectively. The matrices $C$ and $E$ can be easily computed since both basis functions are defined with respect to the same mesh. On the contrary, $D$ and $H$ concern discrete functions living on different meshes. Quadrature formulas, by $N_{q}$ points on the edges on the interfaces $\Gamma$ and $\gamma$, are implemented to compute the terms of $D$ and $H$

$$
\begin{aligned}
& D(i, k)=\sum_{\mathrm{e} \in \Gamma}\left(\sum_{j=1}^{N_{q}} w_{j} \phi_{k}\left(\zeta_{j}\right) \psi_{i}\left(\zeta_{j}\right)\right) \\
& H(i, k)=\sum_{\mathrm{e} \in \gamma}\left(\sum_{j=1}^{N_{q}} w_{j} \psi_{k}\left(\zeta_{j}\right) \phi_{i}\left(\zeta_{j}\right)\right)
\end{aligned}
$$

where $\zeta_{j}$ and $w_{j}$ are the quadrature points and weights. Each point of quadrature of (6) and (7) is located within $\mathcal{T}_{F}$ and $\mathcal{T}_{M}$, respectively.

Fig. 2 (right) shows the computation for an edge $\left[A_{1}, A_{2}\right]$ of the matrix D. For the first quadrature node $P_{1}$, there are three contributions of the element $\left(N_{1} N_{2} N_{3}\right)$ such $\left(w_{1} \phi_{k}\left(P_{1}\right)\left(\psi_{N_{1}}\left(P_{1}\right)+\psi_{N_{2}}\left(P_{1}\right)+\psi_{N_{3}}\left(P_{1}\right)\right)\right)_{k=A_{1}, A_{2}}$. For the second point $P_{2}$ in $\left(N_{2} N_{3} N_{4}\right)$, there are $\left(w_{2} \phi_{k}\left(P_{2}\right)\right.$ $\left.\left(\psi_{N_{2}}\left(P_{2}\right)+\psi_{N_{3}}\left(P_{2}\right)+\psi_{N_{4}}\left(P_{2}\right)\right)\right)_{k=A_{1}, A_{2}}$. For the last point $P_{3} \quad$ in $\quad\left(N_{3} N_{4} N_{5}\right): \quad\left(w_{3} \phi_{k}\left(P_{3}\right)\left(\psi_{N_{3}}\left(P_{3}\right)+\psi_{N_{4}}\left(P_{3}\right)+\right.\right.$ $\left.\left.\psi_{N_{5}}\left(P_{3}\right)\right)\right)_{k=A_{1}, A_{2}}$.

The indices of nodes in $\mathcal{T}_{M}$ belong to the set $I$ for inner and to the set $B$ for boundary nodes. The finite element matrix, of the moving part, $M_{M}$ thus reads

$$
M_{M}=\left(\begin{array}{ll}
M_{\mathrm{II}} & M_{\mathrm{IB}} \\
M_{\mathrm{BI}} & M_{\mathrm{BB}}
\end{array}\right) .
$$

To solve a single algebraic system, the conditions (3) and(4) are imposed, on the global matrix, with the help of Lagrange Multipliers [10]. The approximation of the physical solution is computed by solving the system

$$
\mathcal{M A}=F
$$

The global matrix $\mathcal{M}$ is composed as

$$
\left(\begin{array}{ccccc}
M_{\mathrm{F}} & 0 & 0 & -D^{\mathrm{T}} & E^{\mathrm{T}} \\
0 & M_{\mathrm{II}} & M_{\mathrm{IB}} & 0 & 0 \\
0 & M_{\mathrm{BI}} & M_{\mathrm{BB}} & C^{\mathrm{T}} & \left(H_{\mathrm{I}} M_{\mathrm{II}}^{-1} M_{\mathrm{IB}}-H_{\mathrm{B}}\right)^{\mathrm{T}} \\
-D & 0 & C & 0 & 0 \\
E & 0 & H_{\mathrm{I}} M_{\mathrm{II}}^{-1} M_{\mathrm{IB}}-H_{\mathrm{B}} & 0 & 0
\end{array}\right)
$$

where $M_{\mathrm{F}}$ is the finite element matrix for the fixed subdomain. The nodes, which belong to an element totally covered by $\Omega_{M}$ are not considered in $M_{F}$. The vector of unknowns is defined by

$$
\mathcal{A}=\left(A,\left(A_{M}\right)_{I},\left(A_{M}\right)_{B}, \lambda_{B}, \lambda_{F}\right)^{\prime}
$$

where $\lambda_{B}$ and $\lambda_{F}$ are the Lagrange Multipliers associated to condition (3) and (4), respectively. The right-hand side form (9) reads

$$
F=\left(0,\left(f_{M}\right)_{I},\left(f_{M}\right)_{B}, 0, H_{I} M_{\mathrm{II}}^{-1}\left(f_{M}\right)_{I}\right)^{\prime} .
$$

The resultant system above is not positive definite but symmetric, sparse and can be solved iteratively by a generalized minimal residual algorithm. The main advantage of this approach is the strong coupling between the two subdomains. Indeed, we have a unique linear system to solve for the whole domain. Note that if $\Omega_{M}$ changes position using a rigid movement (as it is generally the case for the electrotechnical applications in 


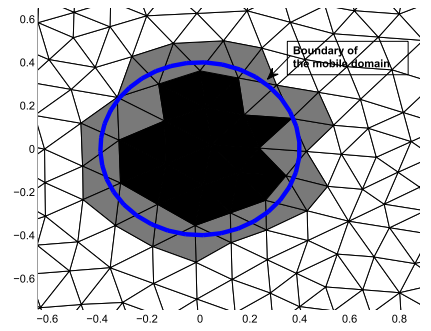

Fig. 3. Treatment of the elements of the fixed domain.

NDT), the only matrices to recompute are $D$ and $H$ since they depend on the position of $\Omega_{M}$ in $\Omega$.

To have a well-posed problem, we need to remove the element in the fixed domain fully covered by the mobile domain [elements in yellow in Fig. 2 (left)]. Indeed, we cannot solve the same problem with two different materials at the same position. It's a major difference between the unidirectional and the bidirectional method.

\section{PRogramming ANd COMPUting Considerations}

The main goal of this method is to fully exploit the domain decomposition approach to reduce the computation time. In this way, the mesh and the matrix for each domain are only computed once before the EC-NDT scan loop. The elementary contributions of the FEM matrix are stored element by element for each domain. At each iteration of displacement, a treatment is necessary only for the fixed part to remove the degrees of freedom attached to the elements fully covered by the moving domain. After having highlighted the elements and their attached nodes, which are fully covered (black) or partially covered (gray) by the moving domain (Fig. 3), an incidence matrix is generated. Each row $i$ of this matrix are associated to the node number. They are initialized to zero. The column $j$ is set to 1 where $j$ is the number of the degree of freedom of the node $i$. The final matrix is obtained multiplying the total matrix by the incidence matrix as follows:

$$
M_{F}^{\text {final }}=P^{t} M_{F}^{0} P .
$$

Each displacement step can easily be distributed on each core or CPU of a computer.

\section{Electrostatic Case}

To evaluate the accuracy of the method, a simple electrostatic case is proposed. An electric scalar potential $V$ is introduced such as the problem to solve reads

$$
\varepsilon_{0} \Delta V=\rho
$$

where $\varepsilon_{0}$ is the dielectric permittivity of free space and $\rho$ is the volumic charge density.

A cylinder with a $1 \mathrm{~m}$ radius is considered. This latter contains the volumic charges of radius equals to $0.2 \mathrm{~m}$. The moving domain is defined by a radius to $0.4 \mathrm{~m}$ and holds in the volumic charges and a box of air Fig. 4. An homogeneous Dirichlet boundary condition is imposed on fixed one. In this configuration, the analytical solution is simple and the created
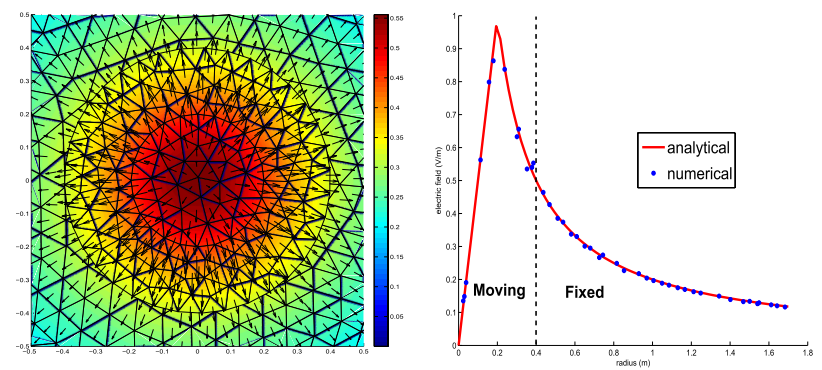

Fig. 4. Focus on the numerical $\mathrm{V}$ and $\mathrm{E}$ solutions on $\Omega_{M}$ (left). Comparison between numerical and analytical solutions of $\mathrm{E}$ according to the radius (right).

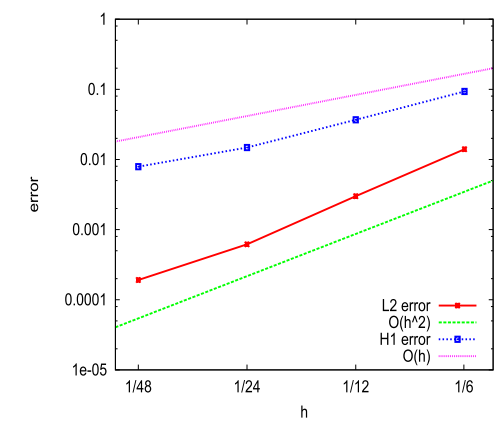

Fig. 5. Convergence rates for the solution in the fixed subdomain.

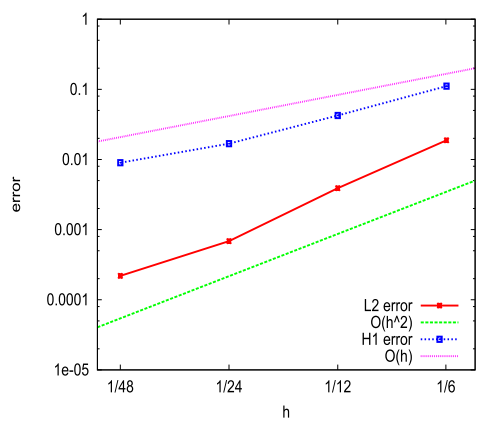

Fig. 6. Convergence rates for the solution in the global solution.

electric field has only a radial component $E$, which can be analytically expressed in terms of radius $r$ by

$$
\vec{E}= \begin{cases}\frac{0.04 \rho}{2 \varepsilon_{0} r} \overrightarrow{u_{r}} ; & r \geq 0.2 \\ \frac{\rho r}{2 \varepsilon_{0}} \overrightarrow{u_{r}} ; \quad r \leq 0.2 .\end{cases}
$$

The performances of the proposed approach can be analyzed. The distribution of the electric scalar potential and field obtained numerically and a comparison between analytical and numerical solutions for the electric field in function of the radial distance are shown in Fig. 4. The solution is continuous through the coupling interfaces.

The spatial errors in $L^{2}$ - and $H^{1}$ - norm, in the numerical solution is determined for different meshes, are represented in a log-log scale in Figs. 5 and 6. Two lines almost parallel implies that the order of convergence are the same for each one. The convergence rates from the numerical to the analytical solution in $L^{2}$ - and $H^{1}$ - norms are in agreement with those predicted theoretically. 


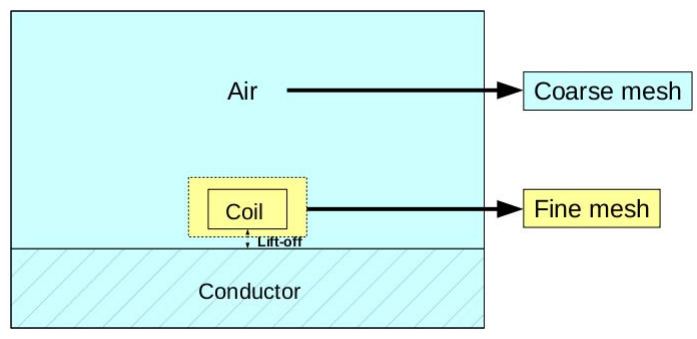

Fig. 7. Domain decomposition into moving and fixed subdomains for EC NDT case.
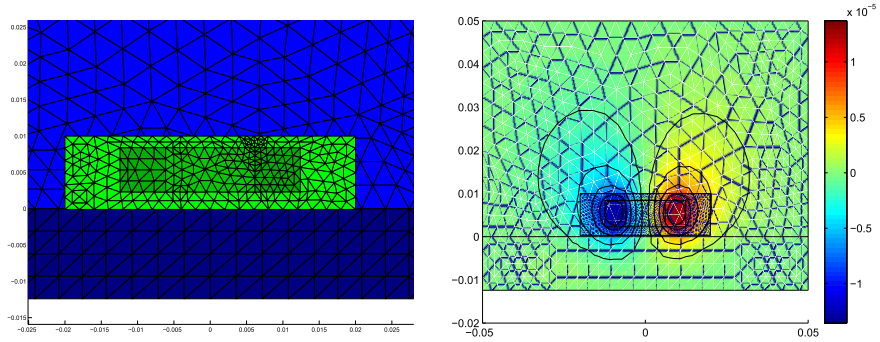

Fig. 8. Focus on moving subdomain in green (left). X-component of magnetic flux density (right).

TABLE I

Relative DEViation BetweEn NumericAl AND MATLAB's PDE SOLUTIONS IN \% FOR DIFFERENT DIMENSIONS OF LIFT-OFF

\begin{tabular}{|c||c|c|c||}
\hline \multicolumn{1}{|c||}{ Lift-off(m) } & $\phi$-FEM $(\mathrm{Wb})$ & $\begin{array}{c}\text { Coil } \\
\phi \text {-Matlab }(\mathrm{Wb})\end{array}$ & Dev $(\%)$ \\
\hline \hline $1.8 \mathrm{e}-03$ & $2.89 \mathrm{e}-05+2.74 \mathrm{e}-06 \mathrm{i}$ & $2.34 \mathrm{e}-05+2.91 \mathrm{e}-06 \mathrm{i}$ & 2.19 \\
\hline $2,0 \mathrm{e}-03$ & $2.31 \mathrm{e}-05+2.64 \mathrm{e}-06 \mathrm{i}$ & $2.35 \mathrm{e}-05+2.79 \mathrm{e}-06 \mathrm{i}$ & 2.13 \\
\hline $2,2 \mathrm{e}-03$ & $2.32 \mathrm{e}-05+2.54 \mathrm{e}-06 \mathrm{i}$ & $2.37 \mathrm{e}-05+2.67 \mathrm{e}-06 \mathrm{i}$ & 2.08 \\
\hline $2,3 \mathrm{e}-03$ & $2.33 \mathrm{e}-05+2.49 \mathrm{e}-06 \mathrm{i}$ & $2.37 \mathrm{e}-05+2.61 \mathrm{e}-06 \mathrm{i}$ & 2.11 \\
\hline
\end{tabular}

\section{EC NDT CASE}

A 2-D EC NDT case is proposed where the problem (2) is solved. The frequency and the lift-off are fixed to $225 \mathrm{kHz}$ and $2.3 \mathrm{~mm}$, respectively. Moreover, we consider $J_{0}=1 \mathrm{~A} / \mathrm{mm}^{2}$, $\sigma=30 \mathrm{MS} / \mathrm{m}$ and $\mu=100 \mu_{0}$ with $\mu_{0}$ the permeability of vacuum.

The magnetic flux density is, mostly, close to the sensor. Thus, the moving subdomain $\Omega_{M}$ contains the coil and a layer of air, as shown in Fig. 7 . We propose to create a fine mesh for $\Omega_{M}$ and a coarse mesh for $\Omega \backslash \Omega_{M}$ Fig. 8 (left).

To determine the accuracy of the method, the flux density is computed Fig. 8 (right) and compared with a MATLAB's Partial Differential Equation toolbox (PDE) toolbox results on a single mesh.

The flux density is continuous across the interfaces of coupling. The magnetic flux $\phi$ in the coil is calculated and the results show a mean difference of $2 \%$ between the MEM and MATLAB's solutions. Henceforth, it is possible to determine the flux for different positions of the coil without remeshing the global domain. Results for different heights of lift-off are shown in Table. I.

Thereafter, an EC NDT case with a defect of size $6.25 \mathrm{~mm}$ in length and $6.1 \mathrm{~mm}$ in depth is considered. Fig. 9 shows the difference of magnetic flux due to the defect

$$
\Delta \phi=\phi-\phi_{0}
$$

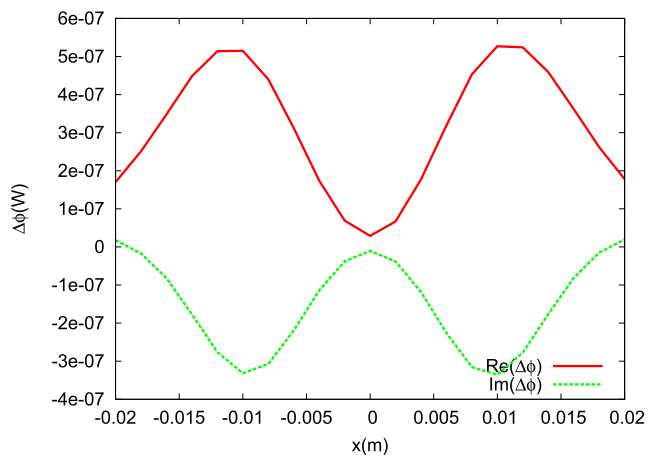

Fig. 9. Variation of the magnetic flux.

where $\phi$ is the magnetic flux with defect and $\phi_{0}$ is the magnetic flux without defect.

\section{CONCLUSION}

The variant of the MEM presented in this paper has the novelty to allow overlapping subdomains with bidirectional exchanges. The order of precision is globally maintained after coupling (in terms of convergence of the error as successive mesh refinements are performed). This paper presents the benefit to allow a strong coupling between the subdomains. In the NDT application, the finite element matrices are evaluated only once at the start of the computation, then the profit in time is proved.

Future works include the extension of this mortar method to an edge element formulation. What's more, the inclusion of a subdomain with different kinds of property will be studied.

\section{ACKNOWLEDGMENT}

This work was supported by CEA LIST.

\section{REFERENCES}

[1] C. R. I. Emson, C. P. Riley, D. A. Walsh, K. Ueda, and T. Kumano, "Modelling eddy currents induced by rotating systems," IEEE Trans. Magn., vol. 34, no. 5, pp. 2593-2596, Sep. 1998.

[2] S. Kurz, J. Fetzer, G. Lehner, and W. M. Rucker, "A novel formulation for 3D eddy current problems with moving bodies using a Lagrangian description and BEM-FEM coupling," IEEE Trans. Magn., vol. 34, no. 5, pp. 3068-3073, Sep. 1998.

[3] D. Rodger, H. C. Lai, and P. J. Leonard, "Coupled elements for problems involving movement,” IEEE Trans. Magn., vol. 26, no. 2, pp. 548-550, Mar. 1990.

[4] I. J. Tsukerman, "Overlapping finite elements for problems with movement," IEEE Trans. Magn., vol. 28, no. 5, pp. 2247-2249, Sep. 1992.

[5] H. Brezis, et al., Nonlinear Partial Differential Equations and their Applications, (Lectures presented at the weekly seminar on applied mathematics), vol. 11. Paris, France: Collège de France Seminar. 1980.

[6] F. Ben Belgacem, "The mortar finite element method with Lagrange multipliers," Numer. Math., vol. 84, no. 2, pp. 173-197, Dec. 1999.

[7] A. Buffa, Y. Maday, and F. Rapetti, "A sliding mesh-mortar method for a two dimensional eddy currents model of electric engines," Math. Model. Numer. Anal., vol. 35, no. 2, pp. 191-228, Mar. 2001.

[8] F. Rapetti, "An overlapping mortar element approach to coupled magneto-mechanical problems," Math. Comput. Simul., vol. 80, no. 8, pp. 1647-1656, Apr. 2010.

[9] Y. Maday, F. Rapetti, and B. I. Wohlmuth, "Mortar element coupling between global scalar and local vector potentials to solve eddy current problems," in Numerical Mathematics and Advanced Applications, F. Brezzi, A. Buffa, S. Corsaro, and A. Murli, Eds. New York, NY, USA: Springer-Verlag, 2003, pp. 847-865.

[10] A. Quarteroni, R. Sacco, and F. Saleri, Numerical Mathematics, 2nd ed. New York, NY, USA: Springer-Verlag, 2007. 